\section{Numerical Evaluation of a Porous Tibial- Knee Implant using Gyroid Structure}

\author{
Basma Eltlhawy ${ }^{1 * \odot}$, Noha Fouda², Ibrahim Eldesouky²
}

\begin{abstract}
Background: Porous materials are recommended for orthopedic applications as they eliminate issues of interfacial instability with tissues and reduce mechanical mismatch of the young's modulus.

Objective: The current research provides a finite element analysis (FEA) to investigate porous gyroid Ti6Al4V structure compared to a solid stem model for human tibial-knee implantation of total knee replacement (TKR).

Material and Methods: In this study, the implant proximal portion was designed as porous gyroid Ti6Al4V structure with $500 \mu \mathrm{m}$ pore size. CATIA V5R18 was used for modeling both gyroid and full solid models. Structural analysis was carried out using ANSYS R18.1 to evaluate the implant performance.

Results: After gyroid implantation, the maximum von-Mises stress obtained under the tibial tray was increased to $10.081 \mathrm{MPa}$. Also, the maximum shear stress at the stem/bone interface was reduced to $0.7347 \mathrm{MPa}$. The stress concentration at the stem tip and the bone strain energy were also improved. The minimum factor of safety is 4.6 for the gyroid porous implant. A proof of concept model was additively manufactured successfully with pore size $577.7733 \pm 34.762 \mu \mathrm{m}$.

Conclusion: The results indicated enhanced clinical performance of the porous tibial-knee implant compared to the solid titanium implant via increasing the maximum von-Mises bone stresses and decreasing the maximum shear stress at the bone/ implant interface.
\end{abstract}

Citation: Elthawy B, Fouda N, Eldesouky I. Numerical Evaluation of a Porous Tibial-Knee Implant using Gyroid Structure. J Biomed Phys Eng. 2022;12(1):75-82. doi: 10.31661/jbpe.v0i0.2005-1116.

\section{Keywords}

Total Knee Replacement; Porous; Knee Prosthesis; Titanium Alloy (Ti6Al4V); Von-Mises Stress; Shear Stress

\section{Introduction}

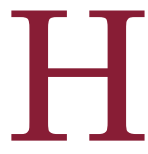

ip and knee replacements improve the patient's life quality via pain relief and restored function, through the success of implants' fixation to bone. Roughened and porous surfaces have been used to improve biological fixation and osseointegration. Recently, porous metals were used to improve fixation to bone for orthopedic applications [1].

Metal lattices fabricated by additive manufacturing (AM) technology with suitable parameters can replicate the biomechanical properties of the natural bone to overcome issues such as bone resorption, implant loosening $[2,3]$. Several researchers have proven the ability of porous materials to substitute natural bone, leading to a promising solution for
${ }^{1} \mathrm{PhD}$ Candidate, Assistant Lecturer, Department of Mechanical Engineering, Higher Future Institute of Engineering and Technology, Mansoura, Egypt

${ }^{2} \mathrm{PhD}$, Department of Production and Mechanical Design Engineering, Faculty of Engineering, Mansoura University, Egypt

*Corresponding author: Basma Eltlhawy

Department of Mechanical Engineering, Higher Future Institute of Engineering and Technology, Mansoura, Egypt

E-mail: eng.b.eltlhawy@ hotmail.com

Received: 20 May 2020 Accepted: 18 July 2020 
load bearing implants and osseointegration improvement $[1,4]$.

Titanium and its alloys are suitable for medical applications due to their biocompatibility, corrosion resistance and mechanical characteristics. However, fully dense metallic biomaterials develop stress shielding and may cause fibrous tissue ingrowth. Development of porous titanium improves the interconnection between implant and human bone to enhance stabilization, and to decrease both the risk of the bone loss and the implant loosening $[5,6]$.

Balancing is a vital demand between biological and mechanical properties by controlling surface roughness, porosity, pore size, shape, and distribution [7, 8]. Finite Element Analysis (FEM) as numerical method that can be used for testing new lattice designs to predict their mechanical properties for meeting specific requirements before fabrication [9].

Open-cellular triply periodic minimal surface (TPMS) structures such as gyroid structure have a vital potential for design of biomedical implants. In this regard, Zulkifli et al. [2] analyzed several structures for metal implants including gyroid structure. Gyroid structures proved biomimicry features to support cell activity with superior mechanical properties comparable to human bone.

Wen et al. [10] evaluated three different unit cell sizes, including 2, 2.5 and $3 \mathrm{~mm}$ of Ti6Al4V gyroid porous scaffolds for bone implant applications via numerical and experimental analyses under compression test. The lattices were manufactured by electron beam melting (EBM) with high porosities in range of 82 to $85 \%$. The results proved that the mechanical properties of the tested scaffolds were comparable to trabecular bone. The elastic modulus and yield strength ranged from 637 to $1084 \mathrm{MPa}$ and 13.1 to $15.0 \mathrm{MPa}$, respectively.

Additionally, Meneghini et al. [3] investigated two different lattices structures of Ti6Al4V, cube and gyroid, for their morphological and mechanical behavior. Five samples of each type porous structures with pore sizes ranged from 300 to $600 \mu \mathrm{m}$ were modeled and analyzed using FEM following the ISO standard (ISO 13314). The porous structures were manufactured by selective laser melting (SLM) process. Morphological characterization of the structures was performed using micro-CT scan, before conducting a compression test to evaluate the mechanical behavior of scaffolds. Cube and gyroid structures with pore size 300 $\mu \mathrm{m}$ presented similar properties to that of natural bone which make them suitable for tissue ingrowth.

Also, Afonso et al. [11] investigated the elastic modulus and the strength through compression tests for two structures of Ti6A14V, gyroids and diamonds manufactured by EBM. The obtained elastic moduli of these porous structures were comparable to each other and the human cancellous bone.

Whereas, Yang et al. [12] fabricated Graded Gyroid cellular structures (GCSs) using selective laser melting (SLM). Compression tests were carried out to investigate the surface morphology and mechanical response of these lattices. Results indicated that GCSs with density gradient perpendicular to the loading direction had deformation behaviors compared to uniform ones, while GCSs with density gradient parallel to the loading direction had layer-bylayer deformation and collapse performance.

The current research present data that could assist the clinical decision using Ti6Al4V gyroid structure for tibial implant design for total knee arthroplasty (TKA). It's expected that a porous gyroid stem will deliver lower stem pain and micro-motion with favorable stress distribution compared to fully solid stem.

\section{Material and Methods}

This analytical study of tibial-knee implant of solid and porous stem structure design predicts their mechanical response. First step involved creating 3D geometries of solid and porous implants implanted in natural human bone using CATIA software (V5R18, Das- 
FEA of a Gyroid Tibial-Knee Implant

sault Systèmes, Vélizy-Villacoublay, France). For the second step, both solid and porous models were modeled using software ANSYS (Release 18.1, ANSYS, Inc., Canonsburg, PA, USA).

\section{Model geometry}

a. Human Bone

A three-dimensional 3D model of a normal left human tibia bone was used to represent the bone geometry. A flat bone osteotomy was prepared in the upper cortical bone to match the flat surface of the tibial tray, and a cavity was cut in the cancellous bone to accommodate the tibial stem as shown in Figure 1a.

b. Solid Implant Model

The full solid implant was designed for cementless fixation method with two components, as shown in Figure 1b. Ti6Al4V tibial implant was integrated with Ultra High Molecular Weight Polyethylene (UHMWPE tibia tray). The thickness of the tibial tray was $4 \mathrm{~mm}$ with $2 \mathrm{~mm}$ inner groove to house the UHMWPE insert. The proximal stem portion was $40 \mathrm{~mm}$ in length and $12 \mathrm{~mm}$ in diameter. The thickness of the UHMWPE insert was 8 $\mathrm{mm}$ [13].

\section{c. Porous Implant Model}

The porous implant also was designed with the same shape and dimensions of the full solid model. The only difference is the implant stem. The full solid stem was replaced by gyroid porous scaffolds, as shown in Figure 1c. The gyroid unit cell has a $500 \mu \mathrm{m}$ pore size and $200 \mu \mathrm{m}$ strut thickness [2].

\section{Loading Conditions}

The two implants, including solid and gyroid, were subjected to a $2000 \mathrm{~N}$ vertical applied load representing a stance phase during normal walking. The distributed forces were $40 \%$ on the lateral condyle $(800 \mathrm{~N})$ and $60 \%$ on the medial condyle $(1200 \mathrm{~N})$. The distal end of the tibia was fixed in all directions [14]. Figure $2 \mathrm{a}$ shows the loading conditions applied to the models.

The coefficient of friction between the human bone and the implants was set to 0.4 [15]. Material properties were assigned to bone and implant as follow: cortical bone, $\mathrm{E}=17 \mathrm{GPa}$ and $v=0.3$ [16]; cancellous bone, $\mathrm{E}=350 \mathrm{MPa}$ and $v=0.25$ [16]; UHMWPE, $E=1 \mathrm{GPa}$ and $v=0.3$ [14]; and Ti6Al4V alloy $E=110 \mathrm{GPa}$ and $v=0.33[14]$.

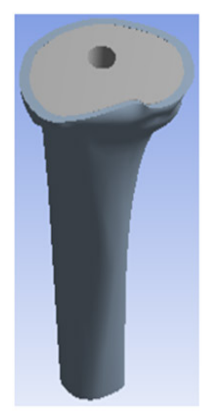

(a)

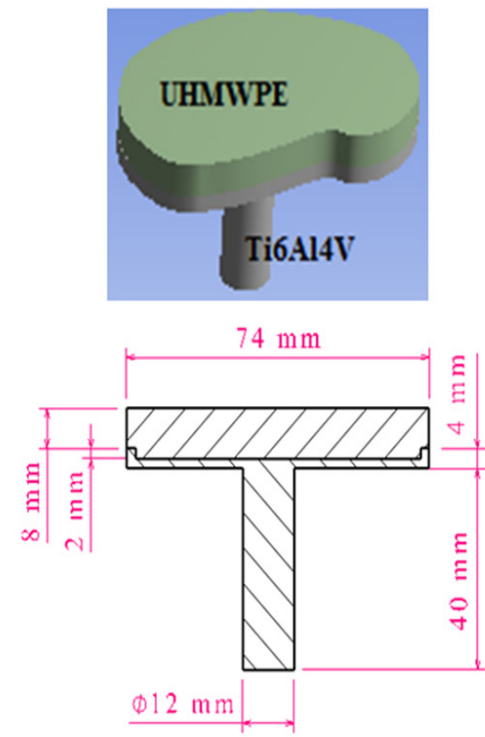

(b)
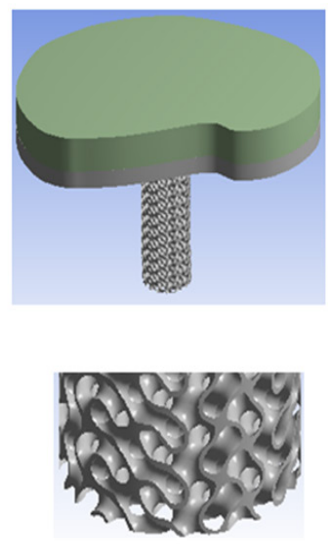

(c)

Figure 1: (a) Prepared bone to insert artificial implant; (b) Geometry of full solid tibia Implant; (c) Gyroid stem implant. 


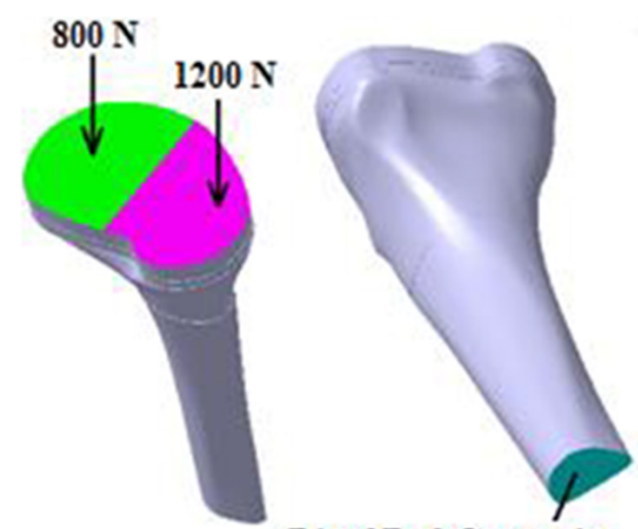

DistalEnd Constraint

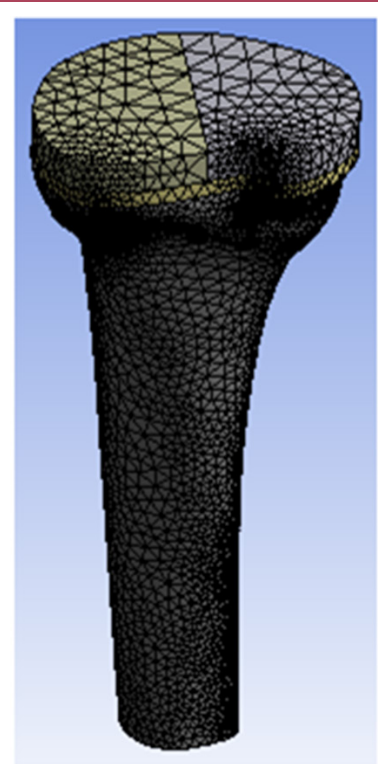

(b)

Figure 2: (a) Loading conditions applied to model; (b) 3D Mesh of bone-implant assembly.

\section{Numerical Modeling}

A 3D finite element analysis was used to study both solid and gyroid implants using ANSYS software (Release 18.1, ANSYS, Inc., Canonsburg, PA, USA). Both solid and gyroid porous models were meshed with solid patch-conforming tetrahedral elements, as shown in Figure 2b. The number of elements of full solid model was 223836 . However, the porous model was tessellated to 313090 elements. The average skewness of the full solid model was 0.26 and the average skewness of the porous model was 0.62 .

\section{Results}

Stress shielding, loosening, and stress concentration are the most commonly failure reasons after TKR. Stress shielding is a result of mismatching between stiffer implant and adjacent bone tissue $[17,18]$. Aseptic loosening is due to micromotion between implant and bone and leads to implant instability. Also, stress concentrations appear at the stem tip and leads to severe patients' pain [18-20].

As shown in Figure 3, the maximum von-
Mises stresses developed in bone under the tibial tray for both solid and porous cases were 7.7457 MPa and 10.081 MPa, respectively.

Additionally, the maximum shear stress developed in bone/implant interface for both solid and porous cases were $0.84604 \mathrm{MPa}$ and $0.7347 \mathrm{MPa}$, respectively, as shown in Figure 4.

Also, the maximum von-Mises stress developed in bone under the stem tip for both solid and porous cases were 1.5174 $\mathrm{MPa}$ and $0.95229 \mathrm{MPa}$, respectively, as shown in Figure 5.

The strain energy developed in bone for both solid and porous cases were $0.11907 \mathrm{~mJ}$ and $0.22784 \mathrm{~mJ}$, respectively, as shown in Figure 6.

Finally, the maximum von-Mises stress value for porous gyroid implant is $202.11 \mathrm{MPa}$, which is approximately $20 \%$ of the yield strength of Ti6A14V (ultimate strength, 970 $\mathrm{MPa}$ and yield strength, $930 \mathrm{MPa}$ ) [21]. Accordingly, the minimum safety factor (SF) of the porous gyroid implant was 4.6. A proof of concept model was built using Phrozen 


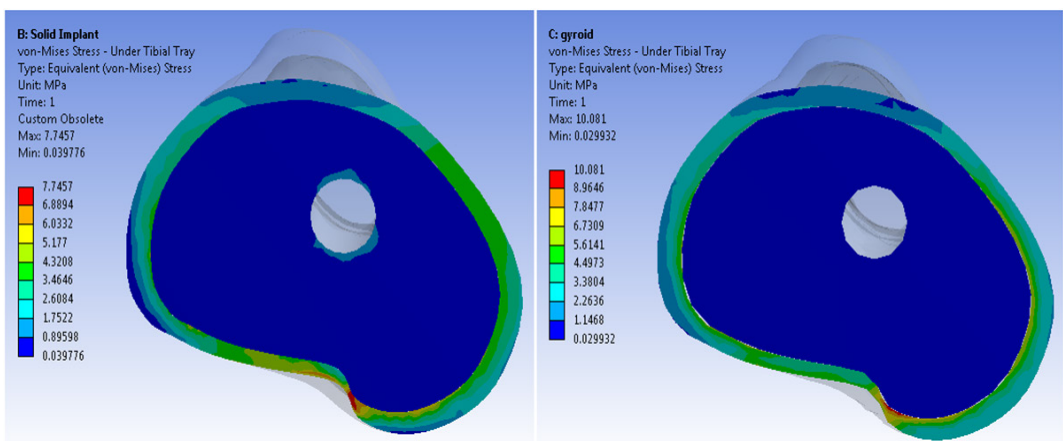

Figure 3: Stress distribution developed in upper surface of bone under the tibial tray for both solid and porous.

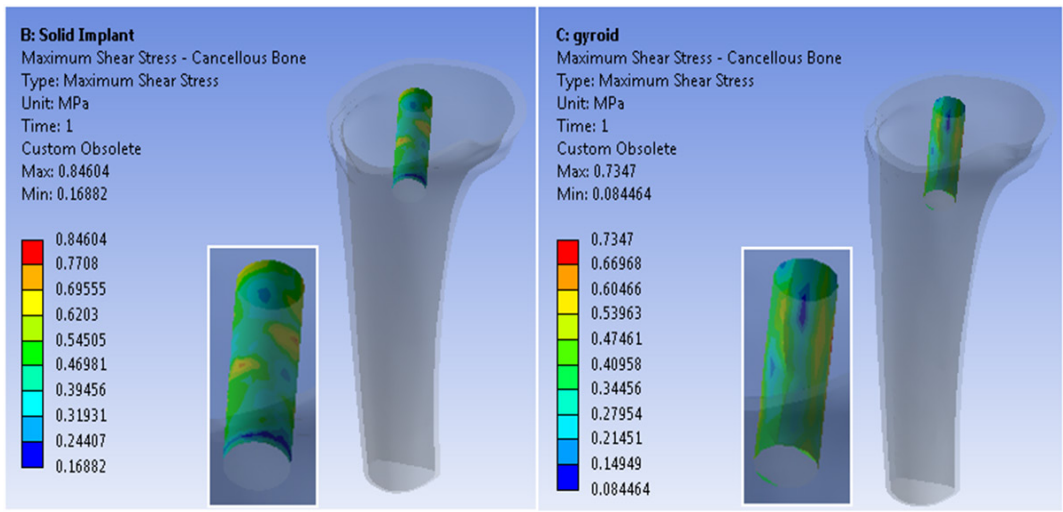

Figure 4: Stress distribution developed in bone/implant interface for both solid and porous cases.

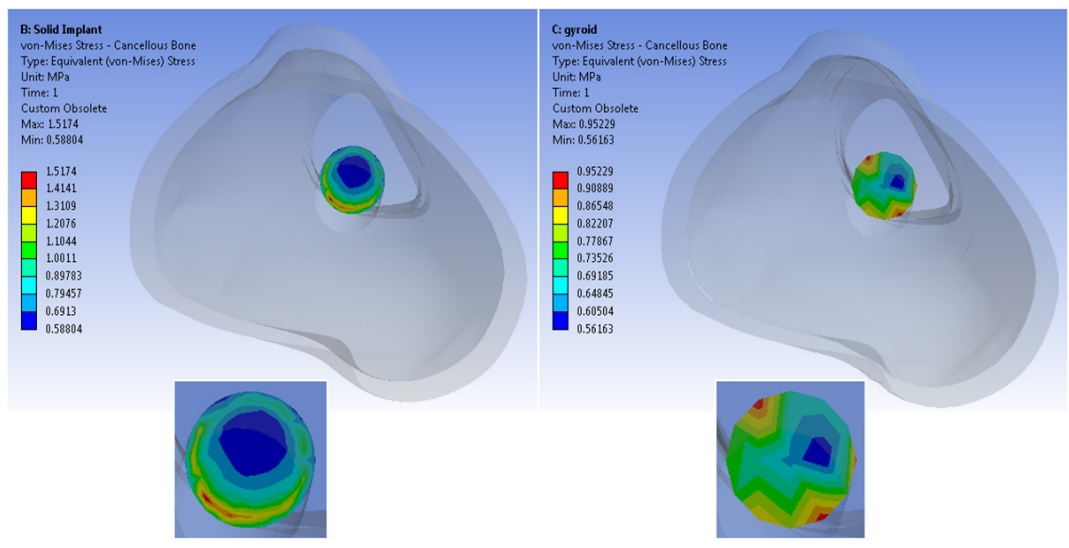

Figure 5: Stress distribution developed in bone under the stem tip for both solid and porous.

Shuffle XL (Phrozen, Hsinchu, Taiwan) as shown in Figure $7 \mathrm{a}$. The average pore size was measured using Quanta FEG-250 Scanning Electron Microscope (Thermo Scientific,
Oregon, USA), Figure $7 \mathrm{~b}$, and was found to be $577.7733 \pm 34.762 \mu \mathrm{m}$, which is acceptable relative to the designed value of $500 \mu \mathrm{m}$ and is within the range suitable for bone ingrowth 


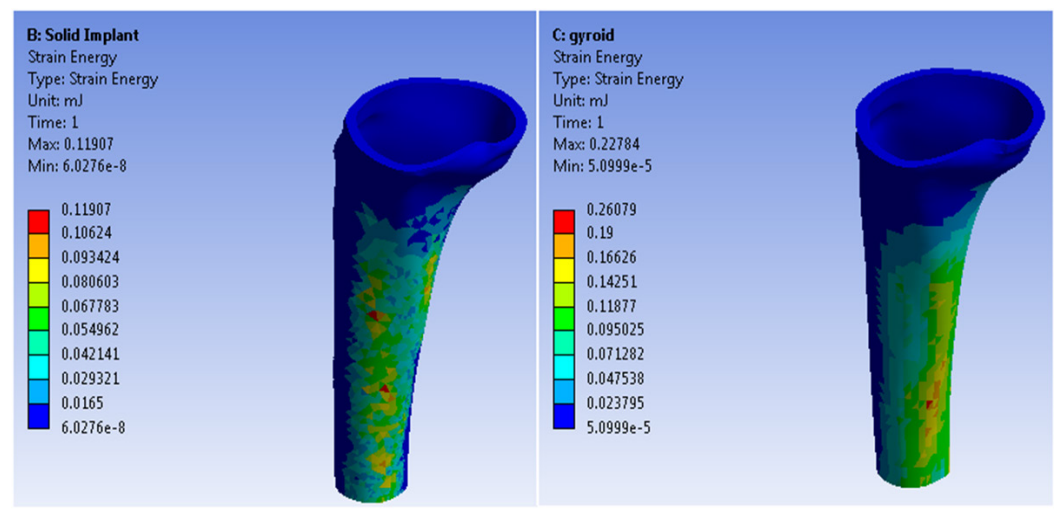

Figure 6: Strain energy distribution developed in the bone for solid and porous.

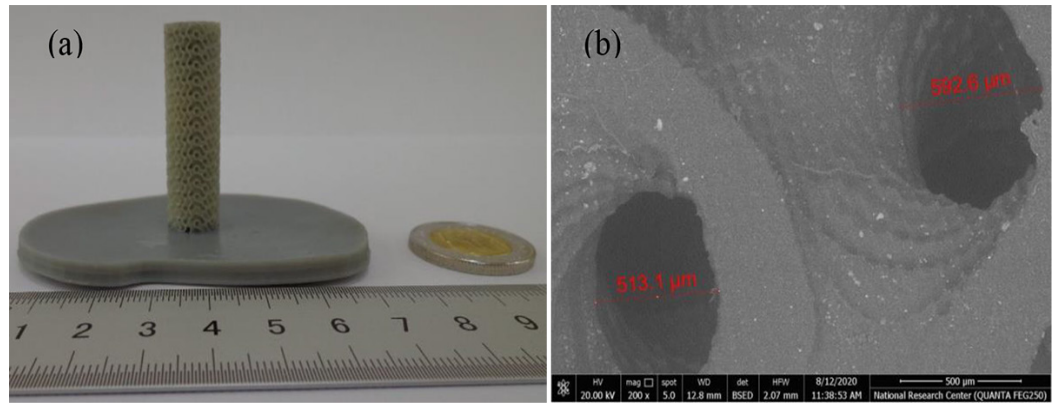

Figure 7: (a) Proof of concept gyroid implant, (b) Scanning Electron Microscope (SEM) image of the pore size.

\section{[3].}

\section{Discussion}

Bone resorption causes clinical and mechanical failure after TKA. The highest probability for bone resorption is under the tibial tray. In this research, gyroid lattice tibial implant was investigated using a numerical analysis in order to compare mechanical behavior against the solid tibial implant under the load applied on the knee.

The results showed a significant increase in maximum von-Mises bone stress under the tibial tray of about $30 \%$ after gyroid implantation compared to solid model. This increase is due to reduction in gyroid scaffolds stiffness to fit the human bone. Therefore, higher stress of gyroid implantation reduces the stress shielding compared to full solid model. Reduction in stress shielding leads to bone resorption re- duction.

Additionally, shear stress along the bone/ stem interface contributes to a mechanical aseptic loosening in the majority of clinical cases. The gyroid scaffolds implant provided $13 \%$ reduction in maximum shear stress along the bone/stem interface.

As stress shielding leads to severe pain besides bone resorption, and the need to relieve this pain is a vital demand. This pain is mostly at the tip of the stem implant, where the stresses concentrated at the cancellous bone and may lead to bone hypertrophy. By comparing stress concentration in bone under the stem tip for both solid and porous implantation, results showed an improved distribution for the gyroid implant. The stress concentration in the stem tip after gyroid implantation reduced by $37 \%$ compared to full solid implantation, as shown in Figure 5. Due to reduction in stress 
concentration value, patient's pain generated within the bone is expected to decrease after gyroid porous implantation compared to solid case.

Strain energy is also a common indicator to judge the performance of implants by comparing the strain energy during loading condition for both full solid and gyroid stem implantation. The results showed an increase in strain energy within the bone after porous implantation about $91 \%$.

The modulus of elasticity and yield strength of human cortical bone range from 4 to $30 \mathrm{GPa}$ and from 20 to $190 \mathrm{MPa}$, respectively [22]. Similarly, the modulus of elasticity and yield strength of human cancellous bone range from 0.2 to $2 \mathrm{GPa}$ and 2 to $80 \mathrm{MPa}$, respectively [22]. The results demonstrated that the stiffness of the gyroid cellular titanium is reduced by approximately $70 \%$ to be comparable to those of human bone, leading to a promising material for load bearing implants. Also, pore size of $500 \mu \mathrm{m}$ and approximately $77 \%$ porosity are suitable for bone ingrowth which increased the bone/implant fixation stability due to enhancing the bone regeneration performance [2]. In addition, the gyroid porous structure can withstand a maximum load capacity of $5000 \mathrm{~N}$ [2].

Patch-conforming tetrahedral mesh was used to obtain a reliable numerical value of stress in the natural tibia to compare with literature [23]. FEA showed a matching between the obtained stresses in natural tibia bone and those in literature [23].

Results prove the possibility of gyroid scaffolds as tailored porosity structures manufactured by additive manufacturing to show promising results for improving tibial-knee orthopedic implants.

\section{Conclusion}

This research depends on a numerical mechanical investigation of a gyroid topology tibial-knee implant to match bone stiffness. The results can be summarized in the follow- ing remarks.

The maximum von-Mises stress under the tibial tray increased approximately by $30 \%$ for gyroid cell model compared to full solid model, leading to less osteopenia.

Also, a micro-motion enhancement is obtained due to reduction in maximum shear stress approximately by $13 \%$ along the stem/ bone interface after gyroid implantation compared to solid state.

In addition, a reduction in stem tip pain is expected due to reduction in stress concentration at the stem tip approximately by $37 \%$ after gyroid scaffolds implantation compared to that of an identical solid titanium implant.

Besides, an increase in strain energy about $91 \%$ is obtained within the bone after gyroid tibial implantation compared to solid case.

Finally, the porous gyroid implant design had 4.6 minimum factor of safety and, the pore size of the proof of concept implant was $577.7733 \pm 34.762 \mu \mathrm{m}$, which is suitable for bone ingrowth.

\section{Acknowledgment}

The software used in this research were generously provided by Egypt-Japan University of Science and Technology (E-JUST). Also, the Scanning Electron Microscope was supplied by The National Research Center.

\section{Authors' Contribution}

Basma Eltlhawy wrote the first draft of the manuscript, analyzed FE, fabricated the samples and conducted experimental tests. Ibrahim Eldesouky edited the paper and provided supervision. Noha Fouda provided supervision. All authors read and approved the final manuscript.

\section{Conflict of Interest}

None

\section{References}

1. Muth J, Poggie M, Kulesha G, Michael Meneghini $R$. Novel highly porous metal technology in artificial hip and knee replacement: Processing methodologies and clinical applications. Jom. 2013;65(2):31825. doi: 10.1007/s11837-012-0528-5. 
2. Zaharin HA, Abdul Rani AM, Azam Fl, et al. Effect of Unit Cell Type and Pore Size on Porosity and Mechanical Behavior of Additively Manufactured Ti6Al4V Scaffolds. Materials (Basel). 2018;11(12):2402. doi: 10.3390/ma11122402. PubMed PMID: 30487419. PubMed PMCID: PMC6317238.

3. Yuan L, Ding S, Wen C. Additive manufacturing technology for porous metal implant applications and triple minimal surface structures: A review. Bioact Mater. 2018;4(1):56-70. doi: 10.1016/j.bioactmat.2018.12.003. PubMed PMID: 30596158 PubMed PMCID: PMC6305839.

4. Banks A. A review of bone implants and the suitability of porous Nitinol. 2014. Available from: http:// faculty.olin.edu/ asieminski/topics/documents/excellent_paper_2008.pdf.

5. Pałka K, Pokrowiecki R. Porous titanium implants: A review. Adv Eng Mater. 2018;20(5):1700648. doi: 10.1002/adem.201700648.

6. Tan XP, Tan YJ, Chow CSL, Tor SB, Yeong WY. Metallic powder bed based 3D printing of cellular scaffolds for orthopaedic implants: A state of the art review on manufacturing, topological design, mechanical properties and biocompatibility. Materials Science and Engineering C. 2017;76:1328-43. doi: 10.1016/j.msec.2017.02.094. PubMed PMID: 28482501.

7. Gao C, Wang C, Jin H, Wang Z, et al. Additive manufacturing technique-designed metallic porous implants for clinical application in orthopedics. RSC Advances. 2018;8(44):25210-27. doi: 10.1039/ c8ra04815k.

8. Wang X, Xu S, Zhou S, Xu W, et al. Topological design and additive manufacturing of porous metals for bone scaffolds and orthopaedic implants: A review. Biomaterials. 2016;83:127-41. doi: 10.1016/j.biomaterials.2016.01.012. PubMed PMID: 26773669.

9. Rotta G, Seramak T, Zasińska K. Estimation of young's modulus of the porous titanium alloy with the use of fem package. Adv Mater Sci. 2015;15(4):29-37. doi: 10.1515/adms-2015-0020.

10. Ataee A, Li Y, Fraser D, Song G, Wen C. Anisotropic Ti6AI4V gyroid scaffolds manufactured by electron beam melting (EBM) for bone implant applications. Mater Des. 2018;137:345-54; doi: 10.1016/j.matdes.2017.10.040.

11. Yánez A, Herrera A, Martel 0, Monopoli D, Afonso $\mathrm{H}$. Compressive behaviour of gyroid lattice structures for human cancellous bone implant applications. Materials Science and Engineering C. 2016;68:445-8. doi: 10.1016/j.msec.2016.06.016. PubMed PMID: 27524040.

12. Yang L, Mertens R, Ferrucci M, Yan C, et al. Contin- uous graded gyroid cellular structures fabricated by selective laser melting: Design, manufacturing and mechanical properties. Mater Des. 2019;162:394404. doi: 10.1016/j.matdes.2018.12.007.

13. Fouda N, Elthawy B, Elmidany T. A three dimensional finite element study for new different knee designs. Trends Biomater Artif Organs. 2017;31(1):9-15.

14. Fouda N, Elthawy B, El-Midany T. The effect of using PLA-HA coating on uncemented tibia prosthesis to decrease aseptic loosening and stress shielding. Int J Mech Mechatron Eng. 2015;15:76-83.

15. Eldesouky I, Harrysson O, West H, Elhofy H. Electron beam melted scaffolds for orthopedic applications. Addit Manuf. 2017;17:169-75. doi: 10.1016/j. addma.2017.08.005.

16. Lai Y-S, Chen W-C, Huang C-H, Cheng C-K, et al. The effect of graft strength on knee laxity and graft in-situ forces after posterior cruciate ligament reconstruction. Plos One. 2015;10:11. doi: 10.1371/ journal.pone.0127293.

17. Gibson LJ. Biomechanics of cellular solids. J Biomech. 2005;38(3):377-99. doi: 10.1016/j.jbiomech.2004.09.027. PubMed PMID: 15652536.

18. Wang Y, Arabnejad S, Tanzer M, Pasini D. Hip implant design with three dimensional porous architecture of optimized graded density. J Mech Des. 2018;140(11):111406. doi: 10.1115/1.4041208.

19. Elthawy $B$. The effect of changing the shape and material of tibial component on the performance of total knee replacement. Mansoura University; 2016. doi: 10.13140/RG.2.1.5134.0400.

20. Shi J. Finite element analysis of total knee replacement considering gait cycle load and malalignment. University of Wolverhampton; 2007. Available from: https://core.ac.uk/download/pdf/1931943.pdf.

21. Eldesouky I, Harrysson 0, Marcellin-Little DJ, West $\mathrm{H}$, El-Hofy H. Pre-clinical evaluation of the mechanical properties of a low-stiffness cement-injectable hip stem. J Med Eng Technol. 2017;41(8):681-91. doi: 10.1080/03091902.2017.1394391. PubMed PMID: 29111845.

22. Li Y, Yang C, Zhao H, Qu S, Li X, Li Y. New Developments of Ti-Based Alloys for Biomedical Applications. Materials (Basel). 2014;7(3):1709-800. doi: 10.3390/ma7031709. PubMed PMID: 28788539. PubMed PMCID: PMC5453259.

23. Mangado N, Quevedo C, Lozano L, Suso S, Cerrolaza $\mathrm{M}$. To what extent the combination of stem length and stem inclination do affect the performance of the tibial component in knee implants? Biomedical Engineering: Applications, Basis and Communications; World Scientific; 2015. doi: 10.4015/s1016237215500180. 